\title{
Treatment of bacterial vaginosis with a three day course of $2 \%$ clindamycin vaginal cream: a pilot study
}

Jyoti Dhar, O P Arya, D J Timmins, S Moss, S Mukembo, A B Alawattegama, O Williams

\begin{abstract}
Objective-To evaluate the efficacy and safety of a 3 day course of $2 \%$ clindamycin cream in the treatment of bacterial vaginosis.

Design-A prospective, randomised, double blind, placebo controlled study.

Setting-Department of Genitourinary Medicine, Royal Liverpool University Hospital.

Subjects-55 female patients aged 18 years and over, and premenopausal, who spontaneously or after questioning complained of symptoms of bacterial vaginosis.

Results-55 patients were enrolled. 44 patients were evaluable at Visit 1 when among the 23 who received clindamycin cream bacterial vaginosis was not present in $22(95 \cdot 6 \%)$ and only one failed treatment. Of the 21 patients in the placebo group only one $(4 \cdot 8 \%)$ patient was cured and $20(95 \cdot 2 \%)$ were failures. Of the 17 patients evaluable at Visit 2 in the clindamycin group, bacterial vaginosis was not present in $14(82 \cdot 4 \%)$ and had recurred in three. No serious adverse events were noted in either group.

Conclusion-This pilot study provides encouraging evidence of the efficacy and safety of a 3 day course of $2 \%$ clindamycin cream in bacterial vaginosis.
\end{abstract}

(Genitourin Med 1994;70:121-123)

University

Department of

Genito-Urinary

Medicine,

Royal Liverpool

University Hospital,

Prescot Street,

Liverpool L7 8XP

J Dhar

O P Arya

D J Timmins

$S$ Moss

$S$ Mukembo

A B Alawattegama

$\mathrm{O}$ Williams

(Presented as a poster at the Spring Meeting of the Medical Society for the at Zurich, 6-9 May 1993)

Address correspondence to: Dr Jyoti Dhar,

Department of

Genitourinary Medicine,

Royal Hallamshire Hospital,

Glossop Road, Sheffield

S10 2JF, UK.

Accepted for publication 4 November 1993 nidazole on the foetus. The recent reports of association of $\mathrm{BV}$ with preterm birth, ${ }^{2}$ low birth weight, postpartum endometritis ${ }^{3}$ and pelvic infection ${ }^{4}$ have also highlighted the need for alternative treatment, especially in pregnant women.

Clindamycin has excellent activity against anaerobic bacteria ${ }^{5}$ and has been found to be effective in the treatment of $\mathrm{BV}$ when given orally in doses of $300 \mathrm{mg}$ twice daily for 1 week $^{6}$ and when applied intravaginally as a $2 \%$ cream twice daily for 5 days $^{7}$ or once daily for 7 days. $^{8}$ The incidence of systemic side effects is low as only approximately $4 \%$ of the cream is absorbed. ${ }^{9}$

We conducted this study to assess the efficacy and safety of a shorter course (3 days) of $2 \%$ intravaginal clindamycin for the treatment of $\mathrm{BV}$ in a prospective, randomised, double blind, placebo controlled trial.

\section{Patients and methods}

Premenopausal women, over 18 years of age, who either spontaneously or on questioning had symptoms suggestive of BV were included. A diagnosis of BV was made in the presence of malodorous vaginal discharge, vaginal fluid $\mathrm{pH}>4.5$, a fishy amine odour after adding $10 \% \mathrm{KOH}$ to the vaginal fluid and clue cells in the vaginal fluid on microscopic examination. Patients were eligible only if findings on a Gram stain of vaginal discharge were consistent with BV (that is there was a marked decrease or absence of lactobacilli morphotypes and a marked increased in other Gram variable morphotypes).

Exclusion criteria were: known allergy to clindamycin, pregnancy or breast feeding, presence of an intrauterine contraceptive device, women not taking adequate contraceptive measures, history of inflammatory bowel disease or diarrhoea, symptoms suggestive of pelvic infection, genital herpes, cervical or vaginal warts, hysterectomy, history of systemic or vaginal antimicrobial therapy in the previous 2 weeks and the presence of any serious disease and infection such as chlamydia, Trichomonas vaginalis or candida at the base line visit. Women who were likely to menstruate within the following week were also excluded.

Clinical evaluation at each visit included a medical and sexual history, examination of the vulva, vagina, uterus and adnexa, description of vaginal discharge, determination of vaginal fluid $\mathrm{pH}$, test of vaginal fluid for fishy odour after addition of $10 \%$ potassium hydroxide, smear of vaginal fluid for clue cells, gram stain of vaginal fluid, wet mount 
Table 1 The efficacy criteria

Success: (bacterial vaginosis is not present)

1. Absence of symptoms.

2. Gram stain negative.

3. Clue cells absent.

4. Normal vaginal fluid.

5. Vaginal fluid $\mathrm{pH}<$ or $=4.5$.

6. Negative amine odour with $\mathrm{KOH}$.

Improved

Criteria 1 to 3 as above BUT two or less of No. 4-6 are true.

Failure

$\mathrm{BV}$ is present based on the criteria described under 'Patients and Methods'.

Recurrence

Following either success or improvement at the first follow-up evaluation, BV recurred as per criteria described above.

and culture for $T$ vaginalis and culture for candida Neisseria gonorrhoeae and Chlamydia trachomatis.

After obtaining written informed consent, the patients were assigned to one of the two treatment groups in a random, double blind, 1:1 fashion. Each patient received a tube containing either $20 \mathrm{~g} 2 \%$ clindamycin vaginal cream or $20 \mathrm{~g}$ matching placebo cream and three disposable applicators. They were instructed to insert $5 \mathrm{~g}$ of cream high into the vagina at bedtime for three consecutive nights and to return any unused cream at the next clinic attendance. The patients were to be assessed at 7 days (Visit I) and 28 days (Visit II) after the start of treatment.

The patients were requested to abstain from sexual intercourse and to avoid prolonged soaking in a bath until the first followup at day 7 (Visit I), after treatment.

At Visit I, any new symptoms, adverse events, sexual history, administration of medication and concurrent use of other agents was noted. Thereafter, clinical examination and base line tests were repeated. The second follow-up at 28 days (Visit II) after completion of therapy included a similar assessment.

The patients were considered to be evaluable at Visit $I$ if they used the medication as described in the protocol, and had not received any other antibiotics or antifungals (topical or systemic), and had not menstruated within 72 hours of the last application of the protocol therapy. The efficacy criteria are defined in table 1.

Statistical analysis for comparison of cure, improvement, recurrence and failure rates between the two groups was done by Fisher's exact test and was considered to be statistically significant at a $\mathrm{p}$ value of $<0.05$.

\section{Results}

Fifty-five women were enrolled, 45 completed the protocol, 24 in Clindamycin Group and 21 in Placebo Group. Comparison of the two groups for age,

Table 2 Outcome of clindamycin versus placebo at Visit I

\begin{tabular}{lllll}
\hline Group & Success & Improve & Failure & Total \\
\hline Clindamycin & 16 & 6 & 1 & 23 \\
Placebo & 1 & - & 20 & 21 \\
\hline
\end{tabular}

Table 3 Outcome of clindamycin versus placebo at Visit II

\begin{tabular}{lllll}
\hline Group & Success & Improve & $\begin{array}{l}\text { Failure/ } \\
\text { Recurrence }\end{array}$ & Total \\
\hline Clindamycin & 12 & 2 & 3 & 17 \\
Placebo & - & - & 3 & 3 \\
\hline
\end{tabular}

weight, menstrual cycle, parity, number of abortions, bowel habits, history of previous infections or previous episodes of $\mathrm{BV}$ and methods of contraception, did not yield any significant differences.

Table 2 lists the treatment outcomes at Visit 1 when 44 patients were evaluable. The overall success together with improvement rate was $96.6 \%(22 / 23)$ with clindamycin and this was highly significant when compared with the placebo group $(\mathrm{p}<0.001)$. Symptomatic candidal vulvovaginitis requiring treatment was seen in one patient in the clindamycin group. Diarrhoea was reported by one patient in each group. However, both the patients with diarrhoea recovered without any residual effects and did not need any treatment.

In the Clindamycin Group, $C$ trachomatis was isolated from the endocervical swabs of three patients who were accordingly treated for chlamydia infection (with tetracycline) at the follow-up Visit I. The one patient with persistent $\mathrm{BV}$ received a course of metronidazole tablets. A further two patients defaulted. After excluding all these patients, 17 were evaluable at follow-up Visit II. Success or improvement was noted in $14(82.4 \%)$ while $B V$ recurred in three (table 3 ). Of the three patients treated for chlamydia infection and one retreated for persistent $\mathrm{BV}$ at the followup Visit $I$, three (including the one retreated for persistent BV) returned at the follow-up Visit II and all were considered cured.

In the Placebo Group, of the 20 with persistent $\mathrm{BV}, 16$ had symptoms of whom five were also found to be infected with $C$ trachomatis. These five cases were, therefore, treated for chlamydia (with tetracycline) as well as persistent BV (with metronidazole) at follow-up Visit I. All of the remaining 11 received a course of metronidazole tablets. A further two patients (including one success at Visit I) defaulted. After excluding all these patients, only three patients (asymptomatic at Visit I) were evaluable at follow-up Visit II, and they had by then begun to re-experience malodorous vaginal discharge. Results of wet film and gram stain of the vaginal material were once again consistent with the presence of BV (table 3). Of the 16 patients retreated at the follow-up Visit I, eight returned at the follow-up Visit II, seven were considered cured and one patient who had received tetracycline and metronidazole, although asymptomatic, showed evidence of BV.

\section{Discussion}

Various treatment regimens, both oral and topical, have been used for BV. At present the United States Center for Disease Control 
recommends metronidazole, $500 \mathrm{mg}$ orally twice daily for 1 week. ${ }^{10}$ (Because of metronidazole formulation, the dose in Great Britain is $400 \mathrm{mg}$, twice daily). However, the possibility of side effects necessitates search for an alternative to metronidazole, especially for use in early pregnancy.

Clindamycin has been shown to be effective in the treatment of this condition. In a study by Greaves et al ${ }^{6}$ oral clindamycin ( 300 mg twice daily for one week) was shown to be effective in $94 \%$ of patients with BV. However, $16 \%$ of patients had adverse effects, although these did not necessitate discontinuation of therapy.

Two recent placebo controlled studies have demonstrated the efficacy of topical intravaginal clindamycin in $\mathrm{BV}$. In a dose ranging study by Livengood et $a l,{ }^{7}$ where $2 \%$ clindamycin was compared with $0.1 \%$ and $1 \%$ concentrations of the cream and used twice daily for 5 days, 93-94\% cure rates were achieved. Hillier et $a l^{5}$ also examined the efficacy of $0 \cdot 1 \%, 1 \%$ and $2 \%$ concentrations of clindamycin applied intravaginally once daily for 7 days. While persistent cure was noted in $94 \%$ of patients who used $2 \%$ clindamycin, the efficacy of the lower concentration creams had dropped to $71 \%$ at one month.

Five grammes of $2 \%$ clindamycin vaginal cream daily for one week has been compared with oral metronidazole, $500 \mathrm{mg}$ twice a day, in two recent studies. ${ }^{811}$ Andres et $a l,{ }^{11}$ reported cure or improvement rates of $97 \%$ with clindamycin and $83 \%$ with metronidazole at one week. This difference was not statistically significant. Schmitt et al, ${ }^{8}$ found a $72 \%$ cure rate with clindamycin and $87 \%$ with metronidazole at one week (difference not significant) and $61 \%$ in each group after one month.
The present study demonstrates that a 3 day course of 5 grammes of $2 \%$ clindamycin cream applied vaginally once daily has similar cure rates as a five or a seven day course referred to above. It is also comparable with treatment with metronidazole, and, pending further proof of its efficacy and its safety in pregnancy, is likely to become the preferred treatment in pregnant women. No serious adverse side effects were noted with its use. Other advantages are topical treatment and possibly better patient compliance due to the short course of treatment.

We thank Stella Hughes, Pamela Roberts, Patricia Kirby, Jean Howel for technical assistance and our nursing staff for help in the clinics. The study was sponsored by Upjohn Limited (U.K.).

1 Department of Health. New cases seen at NHS GenitoUrinary Medicine clinics in England, 1990 Annual fig ures, Summary information from Form KC60.

2 Martius J, Eschenbach DA. The role of bacterial vaginosis as a cause of amniotic fluid infection, chorioaminionitis and prematurity-a review. Arch Gynecol Obstet 1990 247:1-13.

3 Watts DH, Krohn MA, Hillier SL, Eschenbach DA Bacterial vaginosis as a risk factor for postcesarean section endometritis. Obstet Gynecol 1990;75:52-8.

4 Hillier S. Clindamycin treatment of bacterial vaginosis. Rev Contemp Pharmacother 1992;3:263-8.

5 Hillier S, Krohn MA, Watts DH, Wolner-Hanssen P Eschenbach D. Microbiologic efficacy of intravaginal clindamycin cream in the treatment of bacterial vagiclindamycin cream in the treatment
nosis. Obstet Gymecol 1990;76:407-13.

nosis. Obstet Gynecol 1990;76:407-13.
6 Greaves WL, Chungafung J, Morris B, Haile A, Townsend JL. Clindamycin versus metronidazole in the treatment of bacterial vaginosis. Obstet Gymecol 1988 72:799-802.

7 Livengood $\mathrm{CH}$, Thomason $\mathrm{JL}$, Hill GB. Bacterial Vaginosis: Treatment with topical intravaginal clindamycin phosphate. Obstet Gynecol 1990;79:118-23.

8 Schmitt C, Sobel JD, Meriwether C. Bacterial vaginosis: treatment with clindamycin cream versus oral metronidazole. Obstet Gynecol 1992;79:1020-3.

9 Borin MT, Powley GW, Tackwell KR, Batts RN, Batts DH. Absorption of clindamycin in bacterial vaginosis patients after intravaginal application of clindamycin phosphate 2\% cream. Clin Pharmacol Ther 1992;51:2.

10 Centres for Disease Control. 1989 STD treatment guidelines. $M M W R ; 38: 36$.

11 Andres FJ, Parker R, Hosein I, Benrubi GI. Clindamycin vaginal cream versus oral metronidazole in the treatment of bacterial vaginosis: a prospective double-blind clinical trial. South Med $¥$ 1992;85:1077-80. 